\title{
Intratrial proactive interference in rats' serial alternation performance in the radial maze
}

\author{
J. S. COHEN, C. STURDY, and M. HICKS \\ University of Windsor, Windsor, Ontario, Canada
}

\begin{abstract}
Rats acquired a serial alternation task in an eight-arm radial maze that was partitioned into four pairs of arms. Each pair was associated with a different distal stimulus. Rats were initially forced to the left or right arm in each pair (the study segment) before being exposed to both arms in each pair (the freechoice or test segment). Only the previously blocked arm of each pair remained baited. Following initial training, proactive interference (PI) was induced by presenting rats with a forced-choice (prestudy) segment containing arm positions opposite those in the subsequent study segment. Such trials generated poorer free-choice accuracy than did trials without a prestudy segment. Forcing rats to both arms in the pair in a prestudy segment produced only transient PI. A slight improvement in rats' free-choice performance was obtained by forcing them to the same arm position, but only when the test segment was delayed by $30 \mathrm{~min}$. Increasing the interval between the prestudy and study segments from 2 to 30 min eliminated PI, but only when free-choice testing was delayed by 2 min rather than by $30 \mathrm{~min}$. These results suggest that intratrial PI in this preparation was primarily due to confusion about which arm position in each pair had been visited during the last forced-choice segment.
\end{abstract}

Rats' spatial working memory has been measured in the delayed alternation task in the enclosed T-maze (Roberts, 1974) and in the elevated, open radial maze (Olton \& Samuelson, 1976). In the delayed alternation task, rats are initially forced to one arm in the T-maze and are then exposed to both arms, at which time only the previously blocked arm remains baited. In the radial maze task, rats either run the complete maze until all arms have been visited (Olton \& Samuelson, 1976) or are forced to a subset of arms (the "study" segment) and are then exposed, following a variable delay, to the complete maze (the freechoice or retention "test" segment; see Beatty \& Shavalia, 1980). The latter, segmented-trial version is analogous to the delayed alternation task in the enclosed T-maze. In both versions of the radial maze task, rats typically display highly accurate performance and only occasionally reenter a previously visited arm during their final choices in a trial. Indeed, rats' retention of arm locations in the study segment remains relatively intact over several hours (Beatty \& Shavalia, 1980; Maki, 1985; Maki, Beatty, Hoffman, Bierley, \& Clouse, 1984). By contrast, their memory for a single arm position in the enclosed T-maze greatly declines over retention intervals (RIs) of only 45 and $120 \mathrm{sec}$ (Roberts, 1974).

As in other working memory tasks, rats' spatial memory for arm position in an enclosed T-maze or for arm locations in the open radial maze is vulnerable to proactive

The present study is based on a paper delivered at the annual meeting of the Psychonomic Society in Washington, DC, November, 1993. The first and second experiments served as honors theses in partial fulfilment for the B.A. honors degrees by the second and third authors, respectively. Correspondence should be addressed to J. S. Cohen, Department of Psychology, University of Windsor, Windsor, ON, Canada N9B 3P4 (e-mail: a77@uwindsor.ca). interference (PI). PI occurs when memories of prior events intrude upon the retention of more recent ones (Postman \& Underwood, 1973). Such interference from arm location(s) visited on a preceding trial (intertrial PI) or prior to the to-be-retained arm location(s) within a trial (intratrial PI) has been found in both types of tasks.

Intertrial PI has been induced by massing trials in the delayed alternation (Grant, 1981) and radial maze tasks (Dale \& Roberts, 1986; Roberts \& Dale, 1981). Grant (1981, Experiment 2), for example, found that decreasing the intertrial interval (ITI) between successive trials in the delayed alternation task from 60 or $30 \mathrm{sec}$ to $0 \mathrm{sec}$ generated steeper retention gradients in the second trial as the RI was increased from 0 to $40 \mathrm{sec}$. Rats also make more reentries during their final choices in the radial maze on later trials within a session even with ITIs as long as $240 \mathrm{sec}$ (Dale \& Roberts, 1986; Roberts \& Dale, 1981). In these latter studies, rats tended to avoid the last four selected arm locations of a previous trial during their first four initial choices on a current trial, another indication that memories from a preceding trial affect subsequent performance. A recent study from our laboratory (Cohen, Reid, \& Chew, 1994) found that increasing ITIs from 2 min to $2 \mathrm{~h}$ eliminated these disruptive effects and decreased tendencies to avoid the last four selected arm locations of a prior trial. In addition, changing either the proximal texture and visual characteristics of arms or the distal landmark cues between trials likewise attenuated the disruptive effects of massed trials.

Intertrial PI effects have been generally attributed to a loss in temporal discrimination among trial events (Roberts \& Dale, 1981). According to this idea, massing trials causes confusion about whether the arms to be visited during later choices on a trial were already visited earlier during that same trial or were visited on preceding trial(s) 
in the session. Increasing the ITI or differentiating trials by changing proximal arm or distal landmark cues between trials apparently diminishes this confusion by helping rats discriminate when an arm was most recently visited. This recency confusion idea is similar to that proposed by D'Amato (1973) to account for monkeys' nonspatial working memory.

Intratrial PI has also been reliably observed in the delayed alternation task (Gordon, Brennan, \& Schlesinger, 1976; Gordon \& Feldman, 1978; Grant, 1980) and in the segmented radial maze task (Hoffman \& Maki, 1986). In the T-maze, rats are first trained on trials consisting of a forced run to one of the arms (the study segment), followed by a free-choice test to both arms. Following this training, rats are periodically presented with two successive forced-choice runs before the free-choice test run. Only the position of the blocked arm in the second (study) run remains baited in the free-choice test run, however. Forcing a rat to an arm position in the first (prestudy) run that is opposite to that in the study run typically decreases its accuracy on free-choice runs with the magnitude of this intratrial PI comparable at 0- and 40-sec RIs (Gordon et al., 1976; Gordon \& Feldman, 1978; Grant, 1980). By contrast, Roberts (1974) found that forcing rats to the same arm location twice produced better delayed alternation performance when both runs were spaced $60 \mathrm{sec}$ apart or four times regardless of spacing conditions. Gordon et al. (1976) also found similar but nonsignificantly better delayed alternation accuracy when rats were forced to the same arm positions on both forced-choice runs. These latter findings show that PI is not produced simply by presenting a prestudy run per se and that repeating the same arm positions on forced-choice runs tends to promote an opposite proactive enhancement (PE) effect.

Hoffman and Maki (1986) developed an analogous segmented-trial task in an eight-arm radial maze. Rats were first trained on trials in which they were forced to enter four arms in the study segment and were then exposed to all arms in the free-choice test segment. Following training, rats received some trials involving two successive forced-choice segments, a prestudy and a study segment, followed by a free-choice test. There were three different types of prestudy segments. One type consisted of four arm locations that were different from, or opposite to, those in the study segment. Another consisted of four arm locations that were the same as those on the study segment. The third type consisted of all eight arm locations. Hoffman and Maki found lower free-choice accuracy in the radial maze when the prestudy segment contained arm locations that were different from the four arm locations that rats had to visit in the study segment. An even greater disruption in free-choice performance was obtained by forcing rats to all arms during the prestudy segment. Unlike results from the delayed alternation paradigm, however, forcing rats to the same arm locations in prestudy and study segments failed to generate any evidence of PE. It should be noted that Hoffman and Maki tested for intratrial PI and PE with 2-h RIs because, as they noted, spatial working memory in the radial maze remains relatively intact over long RIs. Free-choice accuracy in the delayed alternation task in the enclosed T-maze, on the other hand, rapidly declines over RIs as short as $40 \mathrm{sec}$. Thus, PE might be evident in delayed alternation because the more rapid loss of spatial working memory avoids ceiling effects that would otherwise mask the enhancing effect of repeating the same arms in prestudy and study segments.

The question of major concern in the present study was whether the recency confusion explanation of intertrial PI might also account for intratrial PI in rats' spatial working memory. An alternative explanation of intratrial PI, derived from research on pigeons' nonspatial working memory (Grant, 1975; Grant \& Roberts, 1973), is trace decay. According to this model, the memory trace for each sample declines at a decreasing rate over time. Consequently, the amount of PI from a prestudy sample will be inversely related to the difference between the strength of its trace and that of the study sample at the time of the free-choice test (Grant, 1975). Thus, intratrial PI would be expected to become stronger as the interval between the two samples decreases or as free-choice delay increases, or both. Consistent with this idea, Grant and Roberts (1973) found that pigeons were less accurate on delayed matching when the incorrect color sample occurred before the correct one and that reducing the interval between the two samples increased the size of this PI effect.

As Zentall and Hogan (1977) noted, the recency confusion model may predict the same effect of increasing test delays for entirely different reasons. According to the recency confusion model, intratrial PI depends on the relative, rather than absolute, differences between the interval separating the prestudy from the study samples and that separating the study sample and free-choice test. The two models make different predictions, however, about the effect of varying the interval between the two samples. The trace decay model proposes that increasing the interval between the two samples will reduce intratrial PI regardless of test delays. According to the recency confusion model, however, intratrial PI will be maintained as long as the relative difference between the intersample interval and test delays remains constant.

The major goal of the present study was to test each model's predictions of the effects of varying both the interval between prestudy and study segments and the interval between the study and free-choice test segments on intratrial PI in rats' spatial working memory. Zentall and Hogan (1977) suggested a similar strategy for determining the underlying process responsible for intratrial PI in nonspatial (e.g., delayed matching) tasks. They cautioned, however, that inconclusive results could occur if tasks were either insensitive or too sensitive to the effect of increasing RIs. As has already been noted, rats' spatial working memory in the radial maze is relatively unaffected by very long RIs. Such ceiling effects on performance on control trials (i.e., trials that do not involve prestudy segments) might obscure any differential effects resulting from variations of the two intervals on tri- 
als containing two forced-choice segments. Conversely, if rats can retain arm locations from study segments over long RIs, then they might retain the interfering arm locations from prestudy segments as well. If so, reducing the interval between prestudy and study segments might not increase PI. Therefore, before testing each model's predictions about the effects of varying each intersegment interval on intratrial PI, we modified the radial maze task to make it more sensitive to shorter RIs. Experiment 1 of the present study reports our efforts to modify this task to obtain reliable intratrial PI effects over relatively short $(30-\mathrm{sec})$ intervals. Experiment 2 was designed to assess the effects of covarying the two intersegment intervals.

\section{EXPERIMENT 1}

Experiment 1 attempted to replicate Hoffman and Maki's (1986) findings with a modified radial maze preparation that was more sensitive to short $(30-\mathrm{sec}) \mathrm{RIs}$. It should be noted that rats are typically exposed to more enriched environments in the radial maze than in the enclosed T-maze. Consequently, poorer working memory in delayed alternation might result from a relatively impoverished stimulus environment in the T-maze. Moreover, training rats in a radial maze that is largely devoid of distal landmark cues has been shown to yield lower overall accuracy and also to promote adjacent arm choice algorithms during free-choice tests (Suzuki, Augerinos, \& Black, 1980; Zoladek \& Roberts, 1978). Thus, in designing an adequate radial maze task for this experiment, we not only had to restrict the amount of distal stimuli but also had to prevent such response algorithms because the latter could mask PI or PE effects.

To do this, we essentially transformed the radial maze into a serial alternation task. In the typical serial alternation task, the rat is forced to one arm in each of a few T-mazes before it is tested on a free-choice run in each T-maze. The positions of the blocked and unblocked arms are varied between T-mazes both within and across trials to make this a spatial working memory task. Rats easily acquire this task to high levels of accuracy $(>85 \%)$ and display slightly better retention of the arm position they are forced to enter in the last T-maze (Terry, 1992). We partitioned an eight-arm radial maze into four pairs of arms. Each pair was associated with a specific stimulus that was projected onto a screen located between the ends of a pair's arms. The rats were initially and successively forced to the left or right arm of each pair (the study segment), after which they were exposed to both arms of each pair (the free-choice, or test, segment). Following initial training, the rats were exposed on some trials to a prestudy forced-choice segment before being presented with the study segment. Three different types of prestudy segments were used. On one type, the rats were forced to the same arm position as in the following study segment (prestudy-same-arm trials). On the other types, the rats were forced either to the opposite arm position (prestudyopposite-arm trials) or to both arms of each pair (prestudyall-arms trials). Control trials consisted of only study and test segments (no-prestudy trials) and were interspersed among the various prestudy trials. Only the stimulus associated with a specific pair of arms was present when the rat was forced to one of those arms in the prestudy or study segment or had to choose between both arms in the test segment of the trial.

Our task differed from Hoffman and Maki's (1986) preparation in three important ways. First, our rats were exposed to a far more restricted environment of distal stimuli. Second, our rats received a free-choice test only $30 \mathrm{sec}$ rather than $2 \mathrm{~h}$ after completing the study segment. Third, our rats were tested on two arms of a pair at a time rather than being exposed to all eight arms simultaneously.

If the results from the present experiment replicate the findings of Hoffman and Maki (1986), then prestudy trials involving opposite or all arm locations should produce poorer free-choice accuracy than should trials without prestudy segments. Furthermore, intratrial PI should be greater when rats are forced to both arms than when they are forced only to opposite arm locations during prestudy segments. We also wanted to determine whether exposure to the same arm locations on both forced-choice segments might improve free-choice accuracy (i.e., produce $P E$ ) similar to that found in the delayed alternation T-maze task (Roberts, 1974).

\section{Method}

Subjects. Eight male Long-Evans hooded rats (purchased from Charles River Breeding Farms, St. Constant, Quebec) were used. They were veterans of a previous study in the same radial maze with different distal stimuli (Cohen et al., 1994) and were approximately 250 days old at the beginning of this experiment. They were housed in individual holding cages during the experimental session and in large group cages ( 3 per cage) between sessions. The colony room was kept on a 12:12-h dark:light cycle; experimental sessions occurred during the dark portion of the cycle. Each rat was maintained at $85 \%$ of its free-feeding body weight and was fed $15-20 \mathrm{~g}$ of dry food (Purina Rodent Chow) in its individual holding cage after each experimental session. Water was continuously available in group and holding cages.

Apparatus. The apparatus was an elevated radial maze constructed of aluminum and plastic materials. It stood $70 \mathrm{~cm}$ above the floor in the experimental room and consisted of eight identical arms, radiating $45^{\circ}$ from each other from a central, octagonal choice platform. This choice area was $51 \mathrm{~cm}$ wide, was painted flat black, and was surrounded by a $43-\mathrm{cm}$-high enclosure consisting of eight 13-cm-wide guillotine doors. Each arm was made from channel aluminum $(10 \mathrm{~cm}$ wide $\times 5 \mathrm{~cm}$ deep) and extended $61 \mathrm{~cm}$ from the choice platform. A food cup $(2.5 \mathrm{~cm}$ diam. $\times 2 \mathrm{~cm}$ deep) was placed at the end of each arm. Each arm contained a black metal insert that was level with the choice platform floor and extended out to the food cup. Clear Plexiglas barriers ( $40 \mathrm{~cm}$ high $\times 30 \mathrm{~cm}$ long) were located between arms to prevent the rats from jumping across adjacent arms.

Figure 1 shows a schematic overhead view of the maze within an octagonal enclosure in the running room. As shown in this figure, the maze was enclosed in an octagonal chamber ( $295 \mathrm{~cm}$ wide) made from unpainted wooden panels $(180 \mathrm{~cm}$ high $\times 122 \mathrm{~cm}$ wide $)$. The north, east, south, and west panel each contained a flat white $122 \times$ $122 \mathrm{~cm}$ screen, hung $60 \mathrm{~cm}$ above the floor. The northeast, southeast, southwest, and northwest panels were bare. Half of the northeast panel was hinged like a door to allow the experimenter to enter and leave the maze area. Each arm radiated from the central decision chamber to the left or right edge of the screen-bearing panels, 
such that each screen was positioned beyond and between a pair of arms. Four 35-mm carousel slide projectors (not shown) were located outside and $80 \mathrm{~cm}$ above the enclosure to project white stimuli on a black background onto the screens. The four stimuli were a $109 \times 86 \mathrm{~cm}$ rectangle from a blank slide, a $25 \times 25 \mathrm{~cm}$ diamond centered on the screen, a centered vertical bar $(100 \mathrm{~cm}$ long $\times$ $25 \mathrm{~cm}$ wide), and a group of small $(5-\mathrm{cm})$ squares scattered over the screen. These stimuli were the main source of illumination inside the octagonal enclosure. A video camera built into one of the ceiling light wells, $90 \mathrm{~cm}$ above the maze, provided a complete image of the maze on a TV monitor outside the enclosure. The monitor was located near the north wall of the room in an area that also contained controls for the projectors and the guillotine doors of the maze. The experimenter remained out of the rat's sight at this observing and control station. The room's ceiling lights were off while an animal was in the maze. The only other source of illumination within the room, aside from the projected stimulus on a screen, came from the monitor outside the enclosure.

Procedure. No pretraining, or shaping, procedures were necessary because all rats had had previous training in this maze with different distal stimuli hung on the wall panels (Cohen et al., 1994). The experiment consisted of two phases: an initial training phase consisting of 1 trial per session for 24 sessions, and a prestudy segment phase consisting of 2 trials per session for 18 sessions for a total of 36 trials. Trials in the second phase were separated by a $1.5-\mathrm{h}$ interval. Each arm was baited with three $45-\mathrm{mg}$ dustless food pellets (BioServ, Frenchtown, NJ).

A training trial consisted of two segments: a forced-choice study segment, and the free-choice test segment. A trial began in the darkened room when the rat was placed in the central decision chamber with all guillotine doors down. During the study segment, the rats were exposed to only one of the arms of each pair in a successive clockwise fashion, starting with the south panel. A stimulus was projected only onto the screen that was obliquely exposed to the rat by the exposed arm. A different stimulus was dedicated to each screen, such that the stimuli projected onto the north, south, east, and west panels varied across animals. The sequence of stimuli that each animal received remained the same across segments and trials throughout the experiment. Upon the rat's reentry into the decision chamber from the exposed arm, the stimulus was turned off and the rat had to wait $15 \mathrm{sec}$ before it was exposed to an arm of the next pair with its associated stimulus. Each rat experienced six possible

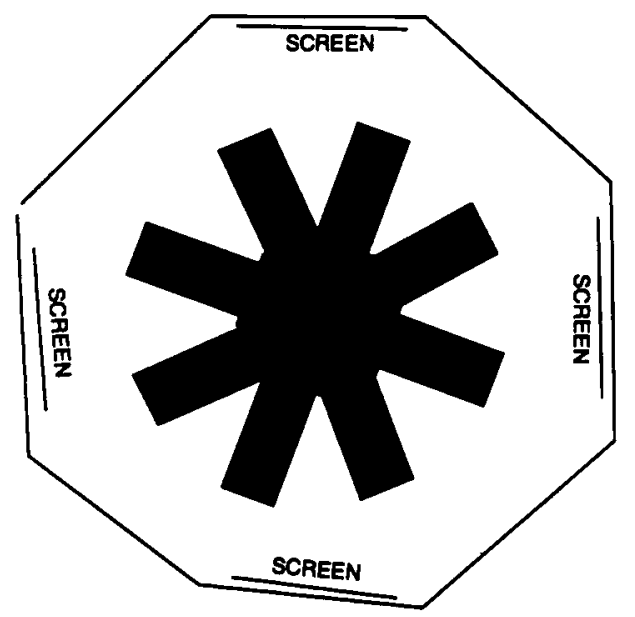

Figure 1. Schematic overhead view of the radial maze and the octagonal enclosure with the screens on the north, east, south, and west panels. The four 35 -mm projectors above and beyond the enclosure and the observation and guillotine control station outside and north of the enclosure are not shown. arm position sequences during the study segment (LRRL, RLLR, RLRL, LRLR, LLRR, and RRLL) on an equal and random basis over the 24 training trials. Following the last single-arm exposure in the study segment, the room lights were dimly illuminated and the experimenter entered the maze area to damp wipe each arm with a weak vinegar solution while the rat waited on the central platform. This procedure took about $30 \mathrm{sec}$. The next (free-choice) test segment of the trial began when the room lights were extinguished. The same stimuli were successively projected onto the screens in the same clockwise fashion, but now both guillotine doors were simultaneously raised to allow the animal to choose either arm of the pair associated with the stimulus. A "correct" choice in each pair was to the previously blocked arm (which remained baited). The same 15-sec delay in the decision chamber between arm pair presentations remained in force. Incorrect, nonreinforced choices were not corrected. All arms and the central chamber of the maze were damp wiped with a weak vinegar solution between animals.

The second (prestudy segment) phase of this experiment was similar to the training phase except that another forced-choice segment occurred prior to the study segment on some trials. Twelve of the 36 trials in this phase contained a prestudy segment in which the rat was successively exposed to both arms in each pair (prestudy-allarms trials), and 12 contained a prestudy segment in which the rat was exposed to only one arm of each pair. For the latter trials, 6 involved exposing the rat to an arm opposite to that presented in the subsequent study segment (prestudy-opposite-arm trials) and 6 were to the same arm (prestudy-same-arm trials). The remaining 12 trials in this phase were control trials that did not contain any prestudy segment (no-prestudy trials).

After completing a prestudy segment, the rat was replaced into its holding cage while the experimenter rebaited and damp wiped the maze for the study and free-choice segments. The room was dimly lit during this short delay of about $45 \mathrm{sec}$. To equalize the amount of food they ingested prior to free-choice (test) segments, the rats were prefed 24 pellets outside the maze prior to their study segment on no-prestudy trials and 12 pellets prior to their prestudy segment on prestudy-same-arm or prestudy-opposite-arm trials. The various types of trials were evenly distributed across sessions to ensure that each occurred equally often as the first trial and as the second trial within a session, with the restriction that the same type of trial never occurred three times in a row (i.e., twice in one session followed by the same type at the beginning of the next session).

Table 1 shows the basic scheme of the intratrial PI phase with examples of each type of segmented trial. Note that trials were organized into two groups: one containing no-prestudy, prestudy-allarms, and prestudy-same-arm trials (prestudy-same-arm/all-arms comparisons), and the other containing no-prestudy, prestudy-allarms, and prestudy-opposite-arm trials (prestudy-opposite-arm/ all-arms comparisons). These groupings allow for comparisons such as those made by Hoffman and Maki (1986). In the prestudysame-arm/all-arms comparisons, 6 prestudy-all-arms trials and 6 prestudy-same-arm trials were compared with their closest 6 noprestudy trials. In the prestudy-opposite-arm/all-arms comparisons, the other 6 prestudy-all-arms trials and the 6 prestudy-opposite-arm trials were compared with those other closest 6 no-prestudy trials.

\section{Results and Discussion}

Training phase. All rats acquired the initial serial alternation task to at least $95 \%$ correct choices within their first 6 trials. Evidence of a slight but reliable serial position effect was observed over the first 12 trials: The rats averaged $89 \%$ correct choices to the first arm pair and averaged more than $95 \%$ correct choices to each of the other pairs $[F(3,21)=5.23, p<.05]$. This effect disappeared, however, over the last 12 trials, during which accuracy was above $95 \%$ correct for each pair. 
Table 1

Examples of Segmented Trials in the Intratrial PI Phase of Experiment 1

\begin{tabular}{|c|c|c|c|c|c|c|c|c|c|c|c|c|}
\hline \multirow[b]{2}{*}{ Trial } & \multicolumn{4}{|c|}{ Prestudy } & \multicolumn{4}{|c|}{ Study } & \multicolumn{4}{|c|}{ Test } \\
\hline & $S$ & W & $\mathrm{N}$ & E & $\mathrm{S}$ & W & $\mathrm{N}$ & E & $S$ & W & $\mathrm{N}$ & $\mathrm{E}$ \\
\hline \multicolumn{13}{|c|}{ Prestudy-Same-Arm/All-Arms Comparisons } \\
\hline No prestudy & & & & & $L$ & $R$ & $R$ & $L$ & $\mathrm{~L} R$ & $L R$ & $L \mathrm{R}$ & $\mathrm{L}$ \\
\hline All arms & $R L$ & $L R$ & $L R$ & $R L$ & $L$ & $R$ & $R$ & $L$ & $\mathrm{~L} R$ & $L R$ & $L R$ & $\mathrm{~L}$ \\
\hline Same arm & $L$ & $R$ & $R$ & $L$ & $L$ & $R$ & $R$ & $L$ & $\mathrm{~L} R$ & $L \mathrm{R}$ & $L \mathrm{R}$ & $\mathrm{L}$ \\
\hline \multicolumn{13}{|c|}{ Prestudy-Opposite-Arm/All-Arms Comparisons } \\
\hline No prestudy & & & & & $L$ & $R$ & $R$ & $L$ & $\mathrm{~L} R$ & $L \mathrm{R}$ & $L \mathrm{R}$ & $\mathrm{L}$ \\
\hline All arms & $R L$ & $L R$ & $L R$ & $R L$ & $L$ & $R$ & $R$ & $L$ & $\mathrm{~L} R$ & $L \mathrm{R}$ & $L \mathrm{R}$ & $\mathrm{L}$ \\
\hline Opposite arm & $R$ & $L$ & $L$ & $R$ & $L$ & $R$ & $R$ & $L$ & $\mathrm{~L} R$ & $L \mathrm{R}$ & $L \mathrm{R}$ & $\mathrm{L} R$ \\
\hline
\end{tabular}

Note- S, W, N, and E define location. Italicized arm positions refer to the baited unblocked arms in the forced-choice prestudy and study segments and to the correct baited arms in the free-choice test segment.

Prestudy segment phase. Figure 2 shows the two sets of comparisons (cf. Table 1) of free-choice performance in our experiment (left panel) and those from Hoffman and Maki's (1986) study. It should be noted that the results of the prestudy-same-arm trials from Hoffman and Maki's second experiment are included with those from their first experiment.

The left panel of Figure 2 shows that the rats in our experiment made fewer correct choices on both prestudyall-arms and prestudy-opposite-arm trials than on noprestudy trials. This intratrial PI effect appeared greater on the prestudy-opposite-arm trials, however. On prestudysame-arm trials, the rats displayed slightly better performance than on no-prestudy trials. The right panel of the figure shows that rats in Hoffman and Maki's (1986) study performed less accurately on prestudy-all-arms trials than on prestudy-opposite-arm trials. Somewhat poorer performance on prestudy-same-arm trials than on no-prestudy trials was also observed, although this difference was not significant.

A three-way, within-subject analysis of variance (ANOVA; number of prestudy arms [one, both, none] $X$ type of comparison [prestudy-same-arm trials vs. prestudy-all-arms trials vs. no-prestudy trials; prestudyopposite-arm trials vs. prestudy-all-arms trials vs. noprestudy trials] $\times$ arm-pair location [south, west, north, east]) was carried out on the free-choice data. NewmanKeuls post hoc tests were conducted when appropriate and statistical significance was maintained at $p=.05$. Analyses of the data shown in the left panel of Figure 2 revealed a significant interaction between number of prestudy arms and type of comparison $[F(2,14)=7.29$, $p<.01]$. Post hoc comparisons indicated that the rats made significantly fewer correct choices on prestudyopposite-arm trials than on their respective no-prestudy trials. The rats also made significantly fewer correct choices on prestudy-all-arms trials than on no-prestudy trials when these data were pooled over both sets, although the difference within each set barely missed significance (critical difference $=8.5 \%$; actual respective differences $=7.3 \%$ and $7.8 \%$ ). The slight improvement on prestudy-same-arm trials relative to their respective no-prestudy trials was not significant.
A slight but significant interaction among all three factors was also uncovered $[F(6,42)=2.52, p<.05]$. This effect apparently resulted from better performance on prestudy-opposite-arm trials across successively presented stimulus locations $(60 \%$ at the south, $64 \%$ at the west, $67 \%$ at the north, and $75 \%$ at the east).

This experiment thus demonstrates intratrial PI over very short test delays in a serial alternation task in the radial maze. Unlike Hoffman and Maki's (1986) study, however, it failed to uncover greater intratrial PI when the rats were forced to both arms than when they were forced only to the opposite arm during prestudy segments. In fact, intratrial PI on prestudy-all-arms trials was not reliably obtained within each set of data. We attempted to resolve the discrepancy between our results and those of Hoffman and Maki (1986) by running our

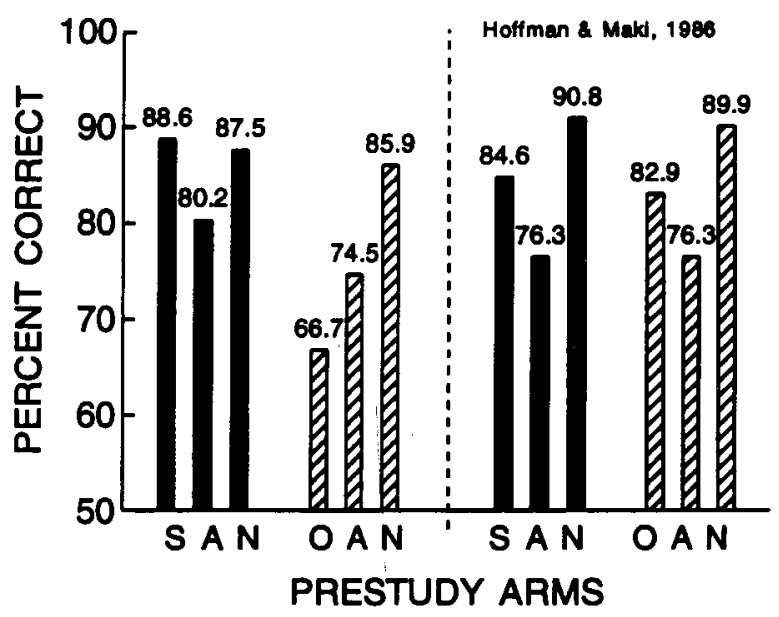

Figure 2. Mean percent correct choices on free-choice segments on prestudy-same-arm (S), prestudy-all-arms (A), prestudyopposite-arm (O), and no-prestudy $(\mathrm{N})$ trials in Experiment 1 in the present study (left panel) and from Hoffman and Maki's (1986) study (right panel) in which data of prestudy-same-arm trials are from their second experiment and data of their noprestudy, prestudy-all-arms, and prestudy-opposite-arm trials are from their first experiment. Data from their prestudy-allarms trials are the same in both comparison groupings to conform to data summary in the present experiment. 
rats on the three types of prestudy trials for another 36 trials in order to determine if the animals would develop poorer accuracy on the prestudy-all-arms trials than on prestudy-opposite-arm trials. Each type of trial was presented equally often in this partial replication. Although the amount of intratrial PI or PE could not be directly assessed because the no-prestudy trials condition was not run in this replication, the rats continued to display the same low levels of free-choice accuracy on the prestudyopposite-arm trials and high levels of free-choice accuracy on the prestudy-same-arm trials, as seen in Figure 2. Of particular interest was the fact that their performance on the prestudy-all-arms trials improved to levels obtained on the prestudy-same-arm trials and on the noprestudy trials seen in Figure 2 . A highly significant effect of prestudy trial $[F(2,12)=41.58, p<.001]$ was obtained that resulted from substantially poorer accuracy on prestudy-opposite-arm trials than on the other two trial types. Thus, the intratrial PI initially observed on prestudy-all-arms trials appeared to be a transient effect in our preparation rather than something that increased with repeated testing (as Hoffman \& Maki's results might suggest).

\section{EXPERIMENT 2}

The results of Experiment 1 indicate that competing information about the locations or position of arms in the prestudy segment of a trial generated substantial intratrial PI in our preparation. The major objective of Experiment 2 was to determine whether the trace decay (Grant, 1975; Grant \& Roberts, 1973) or the recency confusion (D'Amato, 1973; Roberts \& Dale, 1981; Winograd, 1971) explanation better accounts for such intratrial PI. To accomplish this goal, we varied the interval between the prestudy and study segments and between the study and free-choice test segments from 2 to $30 \mathrm{~min}$. Although no evidence of intratrial PE was found on prestudy-samearm trials in Experiment 1, such an effect might have been masked by generally high accuracy on the no-prestudy control trials when tests were delayed only $30 \mathrm{sec}$. Therefore, with the longer test delays in Experiment 2, we again were able to investigate the possibility of intratrial PE.

In Experiment 2, rats received prestudy-same-arm, prestudy-opposite-arm, and no-prestudy trials. Short (2-min) and long (30-min) intervals between the prestudy (or prefeeding on no-prestudy trials) and study segments and between the study and free-choice segments were crossed so that every rat experienced four different interval sequences for each type of trial: a short- short (2$\min -2-\min$ ) sequence, a short-long (2-min-30-min) sequence, a long-short (30-min-2-min) sequence, and a long-long (30-min-30-min) sequence.

Each model makes somewhat different predictions about the effects of varying the two intersegment intervals. According to the trace decay model, the amount of proactive interference (or enhancement) from a prestudy segment is inversely related to the difference between the strength of its trace and that of the study segment at the time of retrieval (Grant, 1975). Given that traces decay at decreasing rates over time, this model predicts that the relative amount of intratrial PI should generally decrease as the interval between the prestudy and study segments is increased to $30 \mathrm{~min}$. This is so because the strength of the competing prestudy traces will have already decayed a considerable amount by the time the rat begins processing the study segment. As the interval between the study and test segment increases, the relative amount of PI should increase as the declining strength of the study segment traces approach those of the prestudy segment. Thus, PI should be most pronounced with a short-long interval sequence and least pronounced with a long-short sequence. Intermediate amounts of PI should be found with the short-short and long-long interval sequences, with much lower amounts for the latter sequence. Thus, the predicted ordering of PI magnitudes would be: shortlong $>$ short-short $>$ long-long $\geq$ long-short.

Although the same reasoning applies in predicting the relative amounts of intratrial PE on prestudy-same-arm trials, the possibility that rats will maintain very accurate retention of information from the study segment over short (2-min) test delays must be considered. Therefore, intratrial PE would be most likely seen with the shortlong interval sequence.

According to the recency confusion model, PI reflects an organism's inability to remember which event it most recently experienced, so the relative differences, rather than the actual durations, of the intersegment intervals should determine the amount of intratrial PI. Thus, decreasing the interval between the two forced-choice segments relative to the interval between the study and test segments should decrease the rat's certainty about which arm was sampled most recently. According to Winograd (1971), this model proposes that PI is inversely related to the ratio of the amount of time elapsing between the presentation of interfering (prestudy) event and the test to the amount of time elapsing between the presentation of the target (study) event and the test. Thus, little or no PI should occur with the long-short (30-min-2-min) sequence, which generates the largest ratio $(32 / 2=16.0)$. The greatest amount of PI should occur under the shortlong sequence because it generates the lowest ratio $(32 / 30=1.07)$, with somewhat lower but equal amounts of PI under either the short-short or long-long sequence, each of which generates the same ratio $(4 / 2=2.0$ and $60 / 30=2.0$, respectively. Thus, the ordering of PI magnitude predicted by this model is: short-long > shortshort $=$ long-long $>$ long-short. Note that, while both models predict the greatest amount of intratrial PI with the short-long sequence and the least amount with the long-short sequence, only the trace decay model predicts more intratrial PI with the short-short than with the long-long sequence.

Unlike the trace decay model, the recency confusion model makes no specific predictions about $\mathrm{PE}$ as a function of intersegment interval sequence. According to this model, repeating the same information across forcedchoice segments need not improve free-choice perfor- 
mance as long as the rat retains information from either segment. A more recent version of this model (Kendrick \& Rilling, 1986), however, predicts PE because such repetitions will supposedly attenuate any processing of irrelevant external or internal events by the rat.

\section{Method \\ Design. The same rats from Experiment 1 were exposed to the same four stimuli in the same locations as in Experiment 1 but ex- perienced them in different orders over trials. The rats also were re- moved from the maze and placed into their holding cages at the end of the study segment as well as at the end of the prestudy segment. Thus, each intersegment interval was varied while the rat waited in its holding cage. This experiment consisted of 36 two-trial sessions. The 72 trials were randomly and equally divided into no-prestudy, prestudy-opposite-arm, and prestudy-same-arm trials. The rats were prefed 12 food pellets prior to their study segment on no- prestudy trials. The intervals between prestudy (or prefeeding) and study segments and between study and test segments were either 2 min (short) or $30 \mathrm{~min}$ (long), such that each type of trial occurred six times under each intersegment interval sequence (short-short, short-long, long-long, long-short). Only 7 of the 8 rats were ana- lyzed because 1 rat became ill and was unable to complete this ex- periment. Free-choice accuracy was analyzed by a within-subject four-way ANOVA (trial type [prestudy- opposite-arm, prestudy- same-arm, no-prestudy] $\times$ first intersegment interval [ 2 or $30 \mathrm{~min}$ ] $X$ second intersegment interval [ 2 or $30 \mathrm{~min}$ ] $\times$ serial position).}

\section{Results and Discussion}

Figure 3 shows free-choice accuracy on prestudyopposite-arm, prestudy-same-arm, and no-prestudy trials at each intersegment interval sequence pooled over serial positions. As this figure shows, the rats were considerably less accurate on prestudy-opposite-arm trials than on no-prestudy trials under short-short, short-long, and long-long interval sequences but were only slightly less so under the long-short interval sequence. Thus, the long-short sequence appeared to produce the least, if any, amount of PI. A slightly greater amount of PI was observed under the short-short than under the shortlong or long-long interval sequences. That is, the difference in the percentage accuracy between no-prestudy and prestudy-opposite-arm trials was greatest at $22 \%$ under the short-short interval sequence and was nearly the same at $16 \%$ and $15 \%$ under long-long and shortlong interval sequences, respectively. On prestudy-samearm trials, the rats appeared to perform slightly better than on no-prestudy trials under each intersegment interval sequence.

A significant triple interaction among trial type and first and second intersegment interval factors $[F(2,12)=$ $5.05, p<.05]$ was uncovered. Post hoc (Newman-Keuls) tests confirmed that this interaction resulted from significant differences between prestudy-opposite-arm and no-prestudy trials under the short-short, short-long, and long-long sequences but not under the long-short interval sequence. Neither the differences in PI between the short-short and short-long or long-long sequences nor those between the prestudy-same-arm and no-prestudy trials were significant.

Further examination revealed that free-choice accuracy was generally poorer when testing was delayed by $30 \mathrm{~min}$

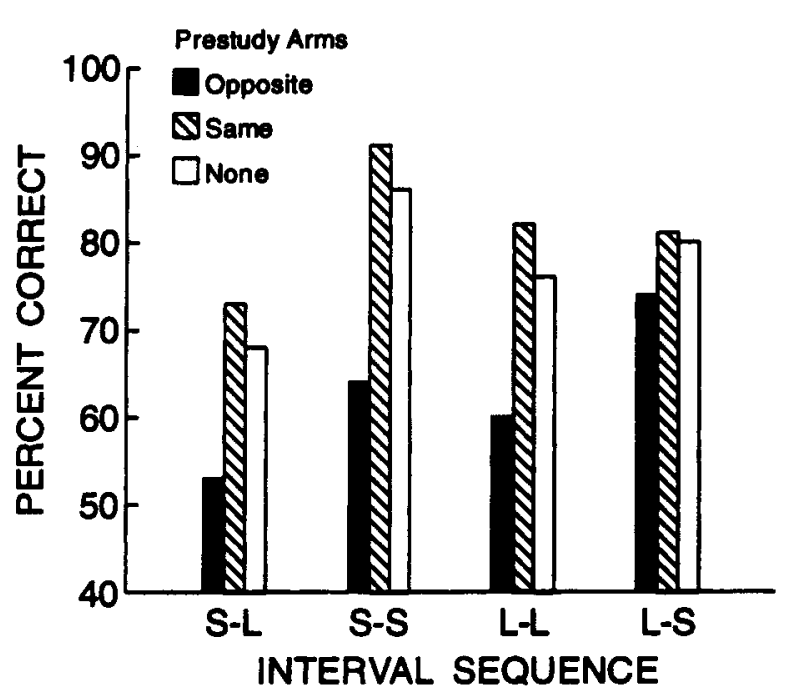

Figure 3. Mean percent correct choices on free-choice tests on prestudy-opposite-arm (Opposite), prestudy-same-arm (Same), and no-prestudy (None) trials as a function of the sequence of interval durations between the forced-choice segments and between the study and free-choice segments in Experiment 2. The sequences are ordered on the basis of the predicted decreasing amounts of PI. S, short (2-min) interval; L, long (30-min) interval.

than when it was delayed by 2 min when the prestudystudy interval was 2 min (compare the short-long interval with the short-short interval). A highly significant double interaction between the first and second intersegment intervals $[F(1,6)=70.94, p<.001]$ and posthoc comparisons supported this observation.

Trial type and the interval between the study and test segments also significantly interacted with serial position of arm-pair presentation $[F(6,36)=4.34, p<.01]$. Figure 4 illustrates this triple interaction. Intratrial PI (i.e., significantly poorer accuracy on prestudy-oppositearm trials than on no-prestudy trials) occurred at the first, third, and fourth serial position when testing was delayed $30 \mathrm{~min}$ and at the first and fourth serial position when it was delayed $2 \mathrm{~min}$. Thus, PI was somewhat more apparent with long test delays than with short test delays. Intratrial PE (i.e., significantly better accuracy on prestudy-samearm trials than on no-prestudy trials) was also obtained, but only when testing was delayed by $30 \mathrm{~min}$. The rats performed significantly better on prestudy-same-arm trials at the second and third serial positions with long delays. Furthermore, free-choice accuracy on these prestudysame-arm trials was significantly better at the second and third serial positions than at the first and fourth serial positions.

Neither model's predictions of the order of magnitude of PI as a function of interval sequence were completely confirmed by this experiment. The general expectation that the short-long sequence would generate the most amount of PI was not borne out. However, failure to obtain this effect could have been due to the significant decline in free-choice accuracy on no-prestudy trials when test delays were increased from 2 to $30 \mathrm{~min}$. As expected, 

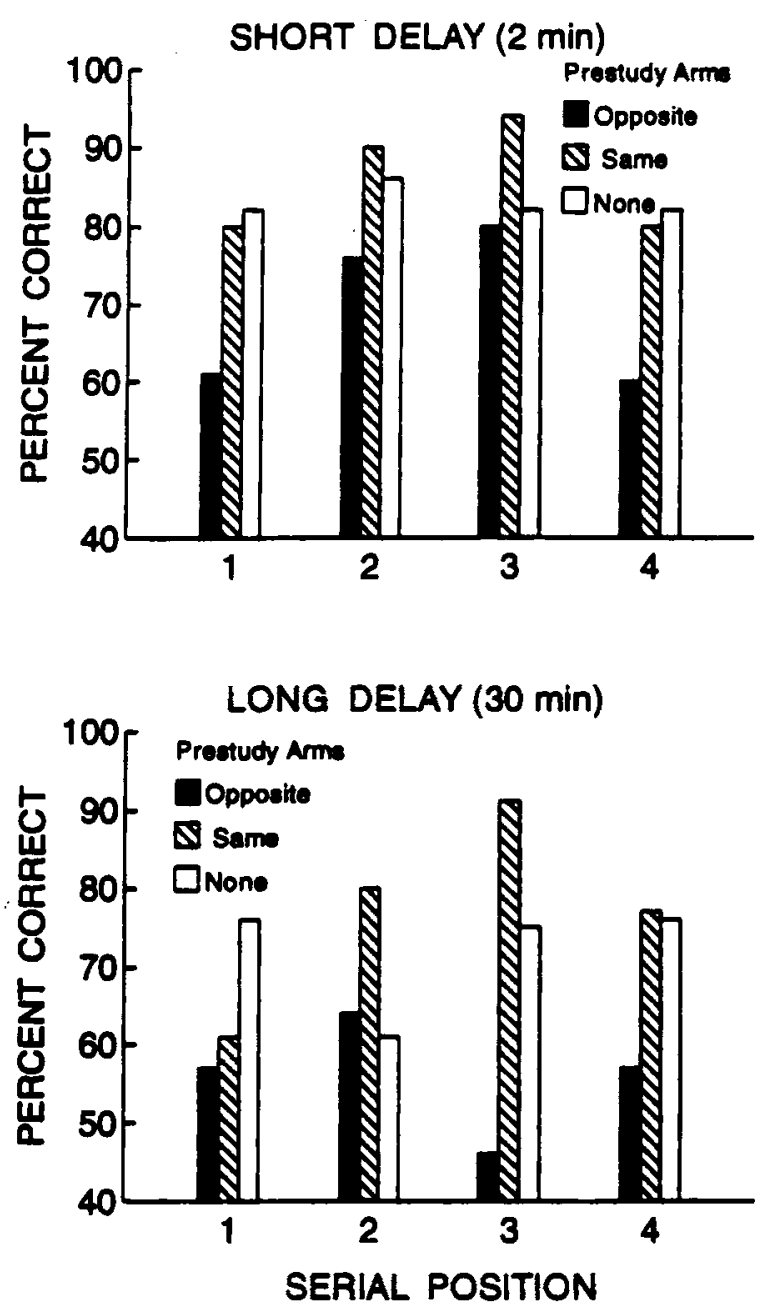

Figure 4. Mean percent correct choices on free-choice segments on prestudy-opposite-arm (Opposite), prestudy-same-arm (Same), and no-prestudy (None) trials at each serial position of the presented stimulus as a function of the delay between the study and free-choice segments in Experiment 2.

little, if any, PI occurred with the long-short sequence. The fact that PI was $6 \%$ greater with the short-short sequence than with the long-long sequence would seem to support the trace decay model rather than the recency confusion model. As already discussed, the latter predicts equal amounts of PI under each interval sequence. A supplementary statistical analysis between these two interval sequences revealed, however, that this difference did not even approach significance $[F(2,12)=0.84, p=$ .49]. Furthermore, the fact that similar amounts of PI occurred under short-long and long-long interval sequences is contrary to the trace decay model. As already noted, this model predicts that PI should be attenuated by the long-long sequence. Therefore, the overall pattern of results and, in particular, the comparable amounts of intratrial PI observed at the short-short, short-long, and longlong sequences are more easily accommodated by the recency confusion account than by the trace decay account.
Some evidence of PE was obtained at the second and third serial positions when test segments were delayed $30 \mathrm{~min}$. This effect appeared to result from an increase in choice accuracy on prestudy-same-arm trials at the middle two serial positions. Both the trace decay model and a more recent version of the recency confusion model (Kendrick \& Rilling, 1986) predict that PE should more likely be seen over longer RIs. The fact that varying the interval between the prestudy and study segments had no effect on PE, however, is more problematical for the trace decay model, which predicts greatest PE at the shortlong interval sequence.

\section{GENERAL DISCUSSION}

The present study accomplished two important goals. First, it demonstrated rats' spatial working memory to be sensitive to intratrial PI in a serial alternation task within the radial maze under much shorter delays than previously used in a more typical radial maze preparation (Hoffman \& Maki, 1986). Second, and more importantly, the results from Experiment 2 indicated that such an effect seems to reflect confusion about which arm locations rats had been forced to visit most recently. Thus, intratrial PI in rats' spatial working memory in this study may be explained by the same temporal discrimination process that was considered to cause intertrial PI in rats' spatial working memory in the T-maze (Grant, 1981) or radial maze (Cohen et al., 1994; Dale \& Roberts, 1986; Roberts \& Dale, 1981).

The methodological implications of Experiment 2 in the present study are especially important in suggesting the necessary conditions required to differentiate between two models of PI. It should be noted that simply varying both the interval between the interfering and to-beretained target information and the interval between the target information and the test for its retrieval may not be sufficient to accomplish this goal. Earlier studies in pigeons' nonspatial working memory for color samples (Grant \& Roberts, 1973; Zentall \& Hogan, 1977), for example, manipulated one or both intervals without conclusively answering this question. Grant and Roberts (1973, Experiment 2) varied the interstimulus interval (0- and 10-sec ISIs) between the first (interfering) and second (sample) stimulus and the retention interval (0and 3-sec RIs) between the sample and test stimuli. Although they found that increasing ISI reduced PI as predicted by either model, increasing RI to $3 \mathrm{sec}$ produced comparably low free-choice accuracy on control and experimental trials. Zentall and Hogan (1977), on the other hand, did not manipulate ISI and found only slight declines in performance when RI was increased from 0 to 5 or 10 sec. Thus, as they pointed out, floor effects in Grant and Robert's (1973) study and ceiling effects in their study prevented any definite conclusion about which underlying process was responsible for intratrial PI. These problems, at least, appear to be avoided when a serial alternation task is adapted for use in the radial maze. 
It should also be noted that the trace decay model cannot be completely dismissed as an account for the results of our Experiment 2. The fact that increasing the interval between prestudy and study segments reduced intratrial PI conforms to either model. As previously discussed, increasing this interval should cause the memories of the interfering information from the prestudy segment to fade according to the trace decay model. According to the alternative recency confusion model, increasing the interval between these successive events reduces PI by helping animals retain their temporal order. Unlike the trace decay model, however, the recency confusion model need not rely exclusively upon the length of time between such events to affect PI. Other nontemporal ways might be used to differentiate successive events to help subjects remember which were more recently experienced. For example, Grant (1980) found that varying the illumination of the T-maze between prestudy and study runs reduced intratrial PI in the delayed alternation task. Apparently, changing contextual stimuli between forced-choice runs promoted better memory of which arm position was last sampled. As previously noted, differentiating trials by changing proximal arm or distal landmark stimuli across massed trials in the radial maze also attenuates intertrial PI (Cohen et al., 1994). Thus, confusion concerning which arms of the radial maze had already been entered during the current or prior trial(s) in a session was reduced by marking trials with these contextual cues. Therefore, the logical next step in our research would be to determine the effects of similar temporal markers of the forced-choice segments on intratrial PI in our segmented radial maze task. According to the recency confusion model, differentiating prestudy and study segments in such a way might eventually override the disruptive effects of decreasing the interval between the forced-choice segments and increasing the interval between the study and free-choice test segments. According to the trace decay model, on the other hand, once the animal has habituated to the novelty of, or has learned the irrelevance of, such contextual cues in determining which arms remain baited during free-choice tests, intratrial PI should increase as the interval between the two forced-choice segments decreases and the RI increases.

In conclusion, findings from the present study and a recent study from our laboratory (Cohen et al., 1994) tentatively suggest that a loss in discriminating the temporal order of successive events may be responsible for intra- and intertrial PI in rats' spatial working memory. This suggestion is in line with evidence from nonspatial working memory tasks that difficulty in discriminating the temporal order of stimuli may be responsible for intertrial PI in those preparations. For example, pigeons' and monkeys' accuracy in determining whether or not a test stimulus occurred as a sample in successive delayed matching-to-sample or as one of the list items in a serialprobe recognition task is poorer when the pool of possible samples or list items is small (Santiago \& Wright, 1984; Wright, Santiago, \& Sands, 1984; Wright, Urcuioli, \&
Sands, 1986). This effect can be attributed to greater confusion about whether an item occurred during a present trial or a previous trial because the items are more likely to be repeated across trials with small pools than with large pools. Indeed, intertrial PI in serial-probe recognition does not occur when lists are composed of novel trialunique stimuli (Wright et al., 1984). More recent research has shown that monkeys' performance in serial-probe recognition declines across sessions, and that such intersession $\mathrm{PI}$ is also eliminated by presenting session-unique stimuli (Jitsumori, Wright, \& Cook, 1988; Jitsumori, Wright, \& Shyan, 1989). Taken together, all of these findings suggest that confusion about when a test item might have occurred can extend across sessions, across trials within a session, and across items within a trial.

\section{REFERENCES}

BeatTy, W. W., \& Shavalia, D. A. (1980). Rat spatial memory: Resistance to retroactive interference at long retention intervals. Animal Learning \& Behavior, 8, 550-552.

CoHEN, J. S., ReID, S., \& CHEW, K. (1994). Effects of varying trial distribution, intra- and extramaze cues, and amount of reward on proactive interference in the radial maze. Animal Learning \& Behavior, 22, 134-142.

DALE, R. H. I., \& RoBERTS, W. A. (1986). Variations in radial maze performance under different levels of food and water deprivation. Animal Learning \& Behavior, 14, 60-64.

D'AMATo, M. R. (1973). Delayed matching and short-term memory in monkeys. In G. H. Bower (Ed.), The psychology of learning and motivation: Advances in research and theory (Vol. 7, pp. 227-269). New York: Academic Press.

GoRdon, W. C., BrenNan, M. J., \& Schlesinger, J. L. (1976). The interaction of memories in the rat: Effects on short-term retention performance. Learning \& Motivation, 7, 406-417.

Gordon, W. C., \& Feldman, D. T. (1978). Reactivation-induced interference in a short-term retention paradigm. Learning \& Motivation, 9, 164-178.

GRANT, D. S. (1975). Proactive interference in pigeon short-term memory. Journal of Experimental Psychology: Animal Behavior Processes, 1, 207-220.

GRANT, D. S. (1980). Delayed alternation in the rat: Effect of contextual stimuli on proactive interference. Learning \& Motivation, 11, 339-354.

GRANT, D. S. (1981). Intertrial interference in rat short-term memory. Journal of Experimental Psychology: Animal Behavior Processes, 7, 217-227.

GRANT, D. S., \& RoBERTS, W. A. (1973). Trace interaction in pigeon short-term memory. Journal of Experimental Psychology, 101, 21 -29.

HoffMan, N., \& MAKI, W. S. (1986). Two sources of proactive interference in spatial working memory: Multiple effects of repeated trials on radial maze performance by rats. Animal Learning \& Behavior, 14, 65-72.

Jitsumori, M., Wright, A. A., \& CoOK, R. G. (1988). Long-term proactive interference and novelty enhancement effects in monkey list memory. Journal of Experimental Psychology: Animal Behavior Processes, 14, 146-154.

Jitsumori, M., Wright, A. A., \& ShYAN, M. R. (1989). Build up and release from proactive interference in a rhesus monkey. Journal of Experimental Psychology: Animal Behavior Processes, 15, 329-337.

KENDRICK, D. F., \& RILling, M. E. (1986). AIM: A theory of active and inactive memory. In D. F. Kendrick, M. E. Rilling, \& M. R. Denny (Eds.), Theories of animal memory (pp. 129-152). Hillsdale, NJ: Erlbaum.

MAKI, W.S. (1985). Differential effects of electroconvulsive shock on concurrent spatial memories: "Old" memories are impaired while "new" memories are spared. Behavioral \& Neural Biology, 43, 162-177. 
Maki, W. S., Beatty, W. W., Hoffman, N., Bierley, R. A., \& Clouse B. A. (1984). Spatial memory over long retention intervals: Nonmemorial factors are not necessary for accurate performance on the radial arm maze by rats. Behavioral \& Neural Biology, 41, 1-6.

Olton, D. S., \& SAMUELson, R. J. (1976). Remembrance of places passed: Spatial memory in rats. Journal of Experimental Psychology: Animal Behavior Processes, 2, 97-116.

Postman, L., \& Underwood, B. J. (1973). Critical issues in interference theory. Memory \& Cognition, 1, 19-40.

ROBERTS, W. A. (1974). Spaced repetition facilitates short-term retention in the rat. Journal of Comparative \& Physiological Psychology, 86, 164-171.

ROBERTS, W. A., \& DALE, R. H. I. (1981). Remembrance of places lasts: Proactive inhibition and patterns of choice in rat spatial memory. Learning \& Motivation, 12, 261-281.

Santiago, H. C., \& WRight, A. A. (1984). Pigeon memory: Samel different concept learning, serial probe recognition acquisition, and probe delay effects on the serial-position function. Journal of Experimental Psychology: Animal Behavior Processes, 10, 498-512.

Suzuki, S., Augerinos, G., \& Black, A. H. (1980). Stimulus control of spatial behavior on the eight-arm maze in rats. Learning \& Motivation, 11, 1-18.
TERRY, W. S. (1992, November). Effects of nonreward in a serial-maze task. Paper presented at the annual meeting of the Psychonomic Society, St. Louis.

WiNOGRAD, E. (1971). Some issues relating animal memory to human memory. In W. K. Honig \& P. H. R. James (Eds.), Animal memory (pp. 259-278). New York: Academic Press.

Wright, A. A., Santiago, H. C., \& Sands, S. F. (1984). Monkey memory: Same/different concept learning, serial-probe acquisition, and probe delay effects. Journal of Experimental Psychology: Animal Behavior Processes, 10, 513-529.

Wright, A. A., UrCuioli, P. J., \& Sands, S. F. (1986). Proactive interference in animal memory. In D. F. Kendrick, M. E. Rilling, \& M. R. Denny (Eds.), Theories of animal memory (pp. 101-125). Hillsdale, $\mathrm{NJ}$ : Erlbaum.

Zentall, T. R., \& Hogan, D. E. (1977). Short-term proactive inhibition in the pigeon. Learning \& Motivation, 8, 367-386.

ZOLADEK, L., \& ROBERTS, W. A. (1978). The sensory basis of spatial memory in the rat. Animal Learning \& Behavior, 6, 77-81.

(Manuscript received November 7, 1994; revision accepted for publication June 6,1995 .) 